\title{
Assessment of Computed Tomography Evaluation Changes in Patients with Acute Pancreatitis
}

\author{
Venkateshwaran Arumugam¹, Sridhar $\mathbf{G}^{2}$ \\ ${ }^{1}$ Associate Professor, Department of Radio-diagnosis, ${ }^{2}$ Senior Resident, Department of Radio-diagnosis, Tagore Medical \\ College and Hospital, India
}

Corresponding author: Dr. Venkateshwaran Arumugam, 16F, Mekalai Appartment, Second Main Road, Annanagar East, Chennai-600102, India

DOI: http://dx.doi.org/10.21276/ijcmsr.2019.4.1.29

How to cite this article: Venkateshwaran Arumugam, Sridhar G. Assessment of computed tomography evaluation changes in patients with acute pancreatitis. International Journal of Contemporary Medicine Surgery and Radiology. 2019;4(1):A113-A115.

\section{A B S T R A C T}

Introduction: Acute pancreatitis is a common disease with a high rate of morbidity and mortality. Study aimed to determine the value of computed tomography evaluation in the early diagnosis of acute pancreatitis.

Material and methods: This prospective study comprises of 60 patients on clinical suspicion/diagnosis of acute pancreatitis, altered biochemical parameters (serum amylase, serum lipase) in favor of acute pancreatitis.

Results: Among these edematous pancreatitis was in $28 \%$ patients and pancreatic necrosis was in $25 \%$ patients. Other features like diffuse/focal pancreatic enlargement in (76\%), peri-pancreatic fat stranding in (63\%) and peri-pancreatic fluid collection in (33\%). Among this alcohol was the most common cause of AP (86\%). The accuracy and sensitivity of serum amylase and serum lipase in diagnosing AP were $45 \%$ and $65 \%$ respectively with CT showing $100 \%$ accuracy and sensitivity. Modified CT severity index was classified as mild ( 2 and 4), moderate (6) and severe (8 and 10) of which majority were mild $(65 \%)$, moderate $(25 \%)$ and severe $(13 \%)$.

Conclusion: Modified CT severity index helps in evaluating the percentage pancreatic necrosis and to predict the possibility of developing local and systemic complications and necessity of tertiary care.

Keywords: Computed Tomography, Acute Pancreatitis, Modified CT Severity Index, Pancreatic Necrosis, Sensitivity.

\section{INTRODUCTION}

Acute pancreatitis is a well known ailment which has a high rate of morbidity and mortality. It possess a huge spectrum of detections that differs in its range from mild or edematous pancreas to ruthless forms having local and systemic complications. The occurrence of this acute pancreatic takes place in $20-30 \%$ of all patients and is featured by an extended clinical course, multiorgan failure and pancreatic necrosis. ${ }^{1}$ The clinical approval or recognition has not been given for the individual laboratory indexes (markers of injury in the pancreas, and the markers of inciting reaction). In order to indicate the severity of the disease the commonly used numerical evaluating systems are RANSON and the APACHE II. ${ }^{2}$ In a heightened condition CT serves as the gold standard method not only for its global picture of the pathology and impediments but also for the non-invasive method of assessing the morphology of pancreas and the peripancreatic areas in a raised moment. It remains unaffected by bowel gas distension and obesity, which for certain is a draw back on ultrasonographic estimation. ${ }^{3}$ The primarily used scoring system was the CT severity index popularly known as the Balthazar Scoring System which was deployed on the pancreatic morphology, the number of peripancreatic fluid collections and the pancreatic necrosis. ${ }^{4}$ The recently Modified Computed Tomography Severity Index (MCTSI) is introduced which varies from the Computed Tomography Severity Index (CTSI). The existence of extra pancreatic impediments and the grading of the peripancreatic fluid accumulation in terms of the presence or absence instead of the number of fluid collections, the grading of necrosis also varies in this system. ${ }^{5}$ Study aimed to assess the CT evaluation and grading of acute pancreatitis

\section{MATERIAL AND METHODS}

This study was carried out in the Department of Radio diagnosis in Tagore medical college and hospital, Chennai from 2018-2019.The study consisted of 60 cases who were diagnosed with acute pancreatitis that is who were clinically believed or doubted to have acute pancreatitis, misrepresented biochemical parameters (serum amylase, and serum lipase) in support of acute pancreatitis. The ultrasonography indicative of acute pancreatitis and a familiar case of chronic pancreatitis with characteristics of acute signs were taken up for computed tomography study and determined.

\section{Inclusion Criteria}

Patients who were doubted or diagnosed having acute pancreatitis based on clinical and biochemical parameters. Patients who were diagnosed of having acute pancreatitis on ultrasonography and also patients who were presented as acute on chronic pancreatitis. 


\section{Exclusion Criteria}

Chronic Pancreatitis.Congenital pancreatic lesion. Pancreatic carcinoma and metastasis. Pancreatic trauma

\section{Study Protocol}

Acquisition of contiguous axial sections, of thickness $5 \mathrm{~mm}$ of abdomen and pelvis, $3 \mathrm{~mm}$ in the region of interest in the craniocaudal track from the point of xiphisternum to pubicsymphysis prior to and subsequent to the administration of oral and intravenous iodinated variation of $80-100 \mathrm{ml}$. The images were viewed in soft tissue window settings. The procedure was clearly explained to the patient and a written consent was obtained from the patient/caretaker. It was instructed that they have to be in overnight NPO and after obtain RFT. All the details related to the clinical, laboratory, ultrasonography and the computed tomography results of the concerned case would be documented as per the proforma. To determine the association (or) the relationship between the two categorical factors Chi square test was enforced. According to the highest score 4 of $43 \%$ and the least score 10 of $1.6 \%$ the patients were distributed to MCTSI.

\section{RESULT}

The patients who underwent an ultrasound prior to $\mathrm{CT}$ were grouped according to normal, direct evidence of pancreatitis and abnormalities consistent with pancreatitis.

Patients were distributed according to MCTSI scores which shows majority in score 4 of $43 \%$ and least in score 10 of $1.6 \%$.

Among these edematous pancreatitides was in $28 \%$ patients and pancreatic necrosis was in $25 \%$ patients. Other features like diffuse/focal pancreatic enlargement in $76 \%$, peri-pancreatic fat stranding in $63 \%$ and peri-pancreatic fluid collection in 33\%. Among this alcohol was the most common cause of AP (86\%). The accuracy and sensitivity of serum amylase and serum lipase in diagnosing AP were $45 \%$ and $65 \%$ respectively with CT showing $100 \%$ accuracy and sensitivity. Modified CT severity index was classified as mild ( 2 and 4), moderate (6) and severe (8 and 10) of which majority were mild (65\%), moderate (25\%) and severe (13\%).

\begin{tabular}{|l|c|c|}
\hline Extrapancreatic complications & No of patients & $\%$ \\
\hline Ascites & 38 & 63.3 \\
\hline Bilateral pleural effusion & 10 & 16.6 \\
\hline Left pleural effusion & 9 & 15 \\
\hline Right pleural effusion & 2 & 3.3 \\
\hline Splenic vein thrombosis & 3 & 5 \\
\hline Portal vein thrombosis & 1 & 1.6 \\
\hline None & 17 & 28.3 \\
\hline \multicolumn{2}{|c|}{ Table-1: Extrapanceratic complication } \\
\hline
\end{tabular}

\begin{tabular}{|l|c|c|}
\hline MCTSI Total score & No of patients & $\%$ \\
\hline 2 & 11 & 18 \\
\hline 4 & 26 & 43.3 \\
\hline 6 & 15 & 25 \\
\hline 8 & 7 & 11.6 \\
\hline 10 & 1 & 1.6 \\
\hline \multicolumn{2}{|c|}{ Table-2: Distribution of patients according to mctsi scores } \\
\hline
\end{tabular}
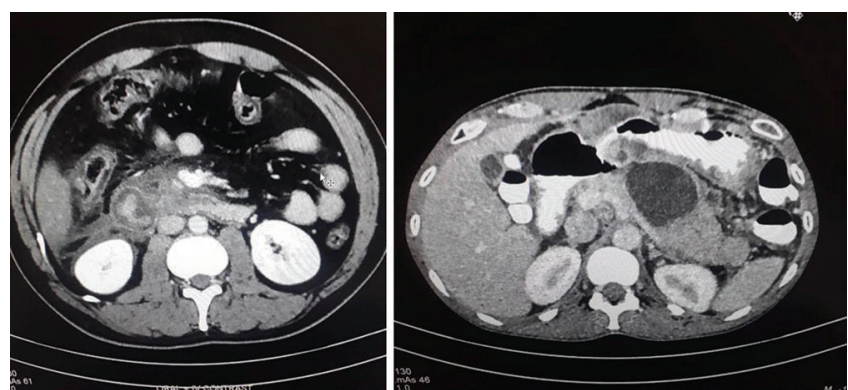

Figure-1: Acute pancreatitis with inflammation extending to duodenum; Figure-2: Acute pancreatitis with pseudocyst

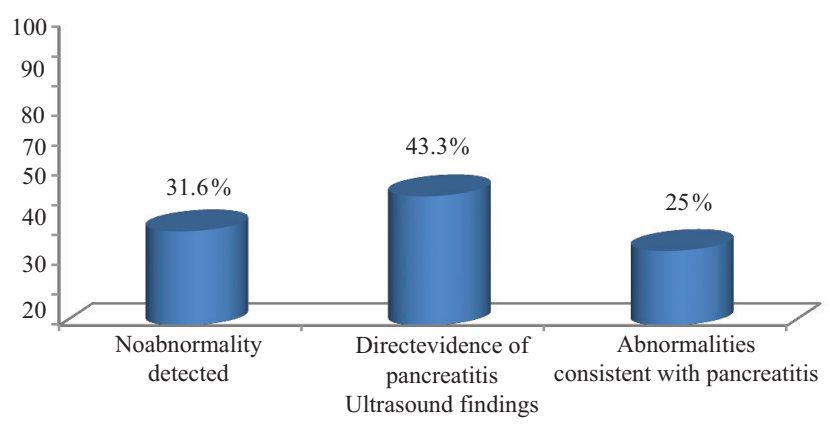

Graph-1: Ultrasound findings

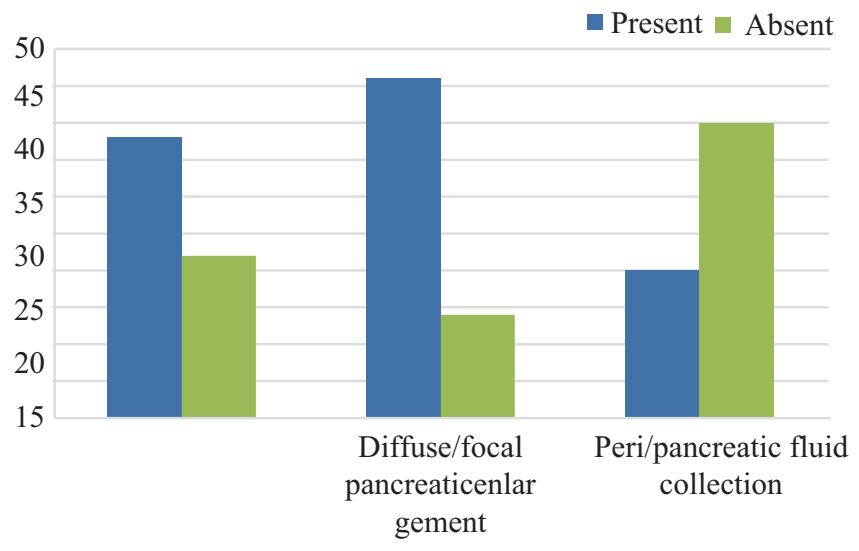

Graph-2: CT findings in ap

\section{DISCUSSION}

Of the 65 cases, 17 (28.3\%) of the patients were found with edematous pancreatitis(fig1), $1525 \%$ were found with pancreatic necrosis out of which 7 had $<30$ of necrosis and 8 had $>30$ of necrosis.(Graph2). As clinical diagnosis alone cannot tell the intensity of the disease CT plays a major role in variating the edematous and necrotizing form of AP. ${ }^{6}$ A study conducted by Beger $\mathrm{H} \mathrm{G}$ et al. and Casas et al. identified necrosis in $18 \%$ and $15 \%$ of the patients with AP respectively and concluded that the occurrence of necrosis happens within $48 \mathrm{hrs}$ after the onset of the symptoms. The detection of Peri-pancreatic fat stranding in normal USG findings, serum amylase and lipase levels suggested the early findings in AP.(Graph1) The pancreatitis in the form of ascites, pleural effusion was observed in 15 patients $(25 \%)$ (Table1). 19(36\%) of the patients showed no abnormality. ${ }^{7}$ Balthazar et $\mathrm{al}^{8}{ }^{8}$ observed abnormal ultrasound findings in $33-90 \%$ of patients with AP. ${ }^{9}$ The edematous pancreatitis was presented as an enlarged hypoechoic gland on ultrasound. 
Thus restricting the role of ultrasound in the imaging of AP in detecting the choleolithiasis and choledocholithasis and the finding of fluid accumulation. The CTs are graded as 2,4,6,8, and 10 as per the MCTSI (Table 2) and are also divided as mild (ranging from 2 and 4), moderate (ranging 6)and severe (ranging from 8 and 10). ${ }^{10}$ The prognosis varied from patients to patients, patients with range 2 and 4 pancreatitis had similar and milder pancreatitis than the patients who were of range $6-10$ in our study. The majority of the patients were between the range of 2 and $4.25 \%$ of the patients showed moderate pancreatitis. ${ }^{11}$ Runzi $\mathrm{M}$ et al. observed that the morphological severity of pancreatitis was determined as mild in 86 (44\%), moderate in 75 (38\%) and severe in 35 (18\%). A study conducted by Chishty et al. in 40 patients revealed that $89 \%$ of them were found with extrapancreatic complication ${ }^{12}, 21(35 \%)$ patients were found with pseudocyst ${ }^{13}$, (Fig2) 2 (3.3\%) of the patients were found with necrosis. ${ }^{12}$ Aspiration of pseudocyst and pleural effusion was needed in $8(13 \%)$ of the patients with range between 4 and 6 of pancreatitis, thus it was concluded and said that patients who needed intervention had more moderate and severe CT grades. ${ }^{14}$ Thus patients who need intervention have more moderate and severe CT grades. ${ }^{15}$

\section{CONCLUSION}

The pancreatic necrosis is evaluated using the MCTSI. As this study was conducted in a rural setting in order to predict the possibility of developing the local and the systemic complications and the need of tertiary care the modified CT severity index was utilized. The development of local and the systemic complications is directly correlated with the MCTSI gradation.

\section{REFERENCES}

1. Banks PA. Practice guidelines in acute pancreatitis. Am J Gastroenterol. 1996; 92(2):377-386.

2. Carl Z, Pallie W. Correlative anatomy and computed tomography: A module on the pancreas and posterior abdominal wall. Radiographics. 1981; 1(3):61-83.

3. Haaga JR, Dogra V, Forsting M, Gilkeson R, Kwon Ha $\mathrm{H}$, Sundaram M. CT and MR imaging of the whole body. 5th ed. Philadelphia: Elsevier; 2003.p.1599-1667.

4. Rizzo R, Szucs R, Turner M. Congenital abnormalities of the pancreas and biliary tree in adults. Radiographics. 1995;15(5):49-68.

5. Kasper D, Braunwald E, Fauci A, Hauser S, Longo D, Jameson L, et al. Harrison's Principles of Internal Medicine. 17th ed. New York: McGraw-Hill; 2008.p.1895-1905.

6. Freeny P.C. Radiology of the Pancreas": Two decades of progress in imaging and intervention. AJR, 1988;150(1):975-981.

7. Beger HG, Rau B, Mayer J, Pralle U. Natural course of acute pancreatitis. World J Surg. 1997;21(5):130-135.

8. Balthazar E. Acute Pancreatitis: Assessment of Severity with Clinical and CT Evaluation. Radiology. 2002;223(6):603-613.

9. Brown A, Baillargeon JD, Hughes MD. Can fluid resuscitation prevent pancreatic necrosis in severe acute pancreatitis? Pancreatology. 2002;2(3):104-7.
10. Kalfarentzos F, Kehagias J, Mead N.Enteral nutrition is superior to parenteral nutrition in severe acute pancreatitis:Results of a randomized prospective trial. Br J Surg.1997;84(1):1665-9.

11. Isenmann R, Runzi M, Kron M. Prophylactic antibiotic treatment in patients with predicted severe acute pancreatitis: A placebo-controlled, double-blind trial. Gastroenterology. 2004;126(4):997-1004.

12. Runzi M, Niebel W, Goebell H. Severe acute pancreatitis: Nonsurgical treatment of infected necroses. Pancreas. 2005;30(1):195-9.

13. Sharma VK, Howden CW. Meta-analysis of randomized controlled trials of endoscopic retrograde cholangiography and endoscopic sphincterotomy for the treatment of acute biliary pancreatitis. Am J Gastroenterol. 1999;94(6):3211-4.

14. Merkle EM, Rich J. Imaging of acute pancreatitis. Eur Radiol. 2002;12(1):1979-1992.

15. Heller SJ, Noordhoek E, Tenner SM. Pleural effusion as a predictor of severity in acute pancreatitis. Pancreas. 1997;15(5):222-225.

\section{Source of Support: Nil; Conflict of Interest: None}

Submitted: 07-02-2019; Accepted: 02-03-2019; Published online: 02-04-2019 